\title{
Exploring the Relationship Between Anxiety and Insistence on Sameness in Autism Spectrum Disorders
}

\author{
Katherine Gotham, Somer L. Bishop, Vanessa Hus, Marisela Huerta, Sabata Lund, Andreas Buja, \\ Abba Krieger, and Catherine Lord
}

\begin{abstract}
Elevated anxiety symptoms are one of the most common forms of psychopathology to co-occur with autism spectrum disorders (ASDs). The purpose of this study was to explore the association between anxiety and ASD symptoms, particularly the degree to which the relationship is explained by insistence on sameness (IS) behaviors and/or cognitive ability. The sample included 1429 individuals aged 5:8-18:0 years who participated in the Simons Simplex Collection, a genetic consortium study of ASD. Child Behavior Checklist Anxiety Problems T-scores and Autism Diagnostic InterviewRevised "IS" item raw totals were treated as both categorical and continuous measures of anxiety and IS, respectively. Chronological age, verbal intelligence quotient (IQ), and a variety of ASD phenotype-related and other behavioral variables were assessed for potential association with anxiety and IS. Anxiety and IS continuous variables were minimally, although significantly, associated with each other and with chronological age and verbal IQ. Neither anxiety nor IS was associated with other core autism diagnostic scores. Anxiety was associated with a variety of other psychiatric and behavioral symptoms in ASD, including irritability, attention problems, and aggression, while IS was not. Anxiety and IS appear to function as distinct constructs, each with a wide range of expression in children with ASD across age and IQ levels. Thus, both variables could be of use in ASD behavioral research or in dimensional approaches to genetic exploration. Unlike IS, however, anxiety is related to non-ASD-specific behavioral symptoms. Autism Res 2013, 6: 33-41. (C) 2012 International Society for Autism Research, Wiley Periodicals, Inc.
\end{abstract}

Keywords: autism spectrum disorders; anxiety; insistence on sameness; Simons Simplex Collection; Child Behavior Checklist 6-18 (CBCL); Autism Diagnostic Interview-Revised (ADI-R)

Autism spectrum disorders (ASDs) are associated with several behavioral features that have genetic and neurobiological implications, including intellectual disability, hyperactivity, aggression, and anxiety. Understanding how ASD and commonly co-occurring symptoms converge and diverge in terms of behavioral, biological, and genetic profiles should contribute new perspectives to the study of autism phenotypes and etiology. For example, these associated features may provide additional means by which to stratify genetic samples (Cannon et al., 2010; Hus, Pickles, Cook, Risi, \& Lord, 2007) or provide insight into basic neurological functioning that could be linked to both ASD and co-occurring disorders. However, before associations with genetic or neurobiological mechanisms can be interpreted, the relationship between behavioral phenotypes of autism and comorbid constructs must be clearly documented in large research samples. The purpose of this study is to explore the association between anxiety and ASD symptoms, particularly the degree to which that relationship is explained by insistence on sameness (IS) behaviors and/or cognitive ability.

Anxiety, whether defined as a constellation of Diagnostic and Statistical Manual of Mental Disorders, Fourth Edition (DSM-IV) or International Classification of Diseases, Tenth Revision disorders or as a psychological trait, is thought to be one of the most common comorbidities with ASD (Howlin, Mawhood, \& Rutter, 2000; Leyfer et al., 2006; Wood et al., 2009). Sukhodolsky et al. (2008) reported that $43 \%$ of an ASD sample $(\mathrm{N}=171)$ from $\mathrm{NIH}$ medication trials met a screening cutoff for at least one

From the Vanderbilt Kennedy Center, Nashville, Tennessee (K.G.); Center for Autism and the Developing Brain, Weill Cornell Medical College/New York Presbyterian Hospital, White Plains, New York (S.L.B., M.H., C.L.); Department of Psychology, University of Michigan, Ann Arbor, Michigan (V.H., S.L.); The Wharton School, University of Pennsylvania, Philadelphia, Pennsylvania (A.B., A.K.)

Received March 12, 2012; accepted for publication October 15, 2012

Address for correspondence and reprints: Katherine Gotham, 1006 Caldwell Lane, Nashville, TN 37204. Email: katherine.gotham@vanderbilt.edu

Conflict of Interest Statement: Dr. C. Lord receives royalties from the publisher of the Autism Diagnostic Interview-Revised (ADI-R) and the Autism Diagnostic Observation Schedule (ADOS), described in this paper. She donates all profits generated by the Simons Simplex Collection use of these measures to charity. Drs. Gotham and Bishop will receive royalties from the ADOS-2, the second edition of the measure described here; both plan to donate all proceeds from research use to charity. Drs. Huerta, Lund, Buja, Krieger, and Ms. Hus have no conflicts of interest.

This work was supported by:

Grant Sponsor: The Simons Foundation (SFARI); Grant Number: Not Applicable (Grants to authors K.G., A.B., and A.K.).

Grant Sponsor: National Institutes of Health; Grant Numbers: T32-MH18921; P30HD15052, R01MH081873-01A1, and RC1MH089721 to C.L.; R01HD065277 to S.B.

Published online 19 December 2012 in Wiley Online Library (wileyonlinelibrary.com)

DOI: 10.1002 /aur. 1263

(C) 2012 International Society for Autism Research, Wiley Periodicals, Inc. 
anxiety disorder. In 177 3- to 18-year-olds with ASD, 25\% received scores in the clinical concern range on the anxiety subscale of the Child Behavior Checklist (CBCL; Achenbach \& Rescorla, 2001) compared with 10\% of their siblings without ASD (Kanne, Abbacchi, \& Constantino, 2009). Additionally, in a study comparing 2323 consecutive referrals to a child psychopharmacology clinic, the 217 children identified with an ASD were at comparable risk for most other psychological disorders, but at significantly increased risk for having two or more anxiety disorders, in comparison with the non-ASD referrals (Joshi et al., 2010).

One explanation for overlap in these symptom families is the hypothesis that common genetic and/or biological factors underlie both ASD and anxiety disorders (e.g. Gadow, Roohi, DeVincent, \& Hatchwell, 2008; Piven \& Palmer, 1999). Evidence of amygdala dysfunction in individuals with ASD (Baron-Cohen et al., 2000; Sweeten, Posey, Shekhar, \& McDougle, 2002) has been suggested as a link to the high rates of anxiety in this population (Amaral, Bauman, \& Schumann, 2003). Studies encompassing a variety of foci for knockout mouse models of autism (including genes involved in serotonin, immunoglobulin, and corticosterone production) have reported both impaired social behavior and increased anxietyrelated behavior in affected mice (Balemans et al., 2010; Benno, Smirnova, Vera, Liggett, \& Schanz, 2009; Lesch \& Mossner, 1998; Takayanagi et al., 2010). Additionally, the elevated rates of anxiety disorders in relatives of individuals with ASD suggest a common genetic link between the two symptom spectra (Bolton, Pickles, Murphy, \& Rutter, 1998; Delong \& Dwyer, 1988; Gadow, DeVincent, \& Schneider, 2008; Piven \& Palmer, 1999; Smalley, McCracken, \& Tanguay, 1995). Although Hallett, Ronald, \& Happé (2009) noted a low level of genetic overlap between anxiety and ASD symptoms in a large study of typically developing twin pairs, they suggest a possible "threshold effect" in which disorders not linked in the general population are linked in clinically affected samples. The question remains whether shared genetic risk between ASD and anxiety disorder explains the high rates of co-occurrence or suggests that anxiety is an intrinsic feature of ASD, possibly as a result of restricted, compulsive behaviors or unusual responses to sensory aspects of the environment (Ben-Sasson et al., 2008; Green \& Ben-Sasson, 2010; Joosten, Bundy, \& Einfeld, 2009).

\section{Anxiety and IS in ASD}

From the earliest history of autism as a defined disorder, Kanner (1943) suggested that restricted and repetitive behaviors (RRBs) were driven by the "anxiously obsessive desire for the maintenance of sameness" (p. 245).

Factor analyses of the Autism Diagnostic InterviewRevised (ADI-R; Rutter, Le Couteur, \& Lord, 2003) and other measures repeatedly yield an "IS" factor (Bishop et al., 2012; Cuccaro et al., 2003; Szatmari et al., 2006) within RRB domains. This factor is characterized clinically by behavioral rigidity and resistance to change. As Kanner suggested, IS behaviors are frequently accompanied by obvious symptoms of anxiety surrounding the maintenance of routines or rituals. In fact, one recent report labeled this the "Anxiety and Compulsions" factor (Kamp-Becker, Ghahreman, Smidt, \& Remschmidt, 2009). However, rates of overlap in anxiety and IS have not yet been documented and replicated in large samples. At this point, it is not established that "anxious" children with ASD tend to exhibit IS or, conversely, that the majority of children with ASD and IS also exhibit anxiety.

\section{The Role of IQ in Anxiety}

Although most of the anxiety-in-ASD literature published to date has been conducted in small samples of individuals with relatively high cognitive abilities, several findings suggest nonetheless that intelligence quotient (IQ) likely plays a significant role in mediating the ASD-anxiety relationship. Sukhodolsky et al. (2008) observed that children with ASD who had high levels of anxiety on the Child and Adolescent Symptom Inventory (Silverman, Fleisig, Rabian, \& Peterson, 1991) tended to have higher IQs and language abilities, as well as more stereotyped behaviors and social impairments on the ADI-R, than did children with lower anxiety and ASD. Another study found that 36 children with ASD and IQ below 70 were significantly less likely to meet criteria for generalized anxiety disorder (GAD) than were a comparison group of 22 children with ASD and IQ of 70 or greater (Witwer \& Lecavalier, 2010). However, the symptom differences appeared to be related to verbally loaded items; physical symptoms of GAD were endorsed at similar rates across the IQ groups. In contrast with the anxiety-IQ findings in ASD, IS has been reported to have minimal association with IQ (Hus et al., 2007), although Cannon et al. (2010) noted that the two were positively correlated when IS factor scores were adjusted to remove the variance shared with repetitive sensory-motor (RSM) behaviors.

In summary, anxiety and ASD commonly co-occur, but it is not clear to what extent their association is explained by IS, a core feature of ASD. Thus, the primary aim of this study is to explore the degree to which anxiety and IS are separable dimensions in ASD. Whereas Kanner (1943) described IS as leading to both anxiety and to RRB, we are starting with the hypothesis that IS and anxiety are separate constructs and testing the association between them to comment on whether anxiety is better described as a component of the ASD phenotype or as a commonly overlapping behavior that is not specific to ASD (see Fig. 1). 


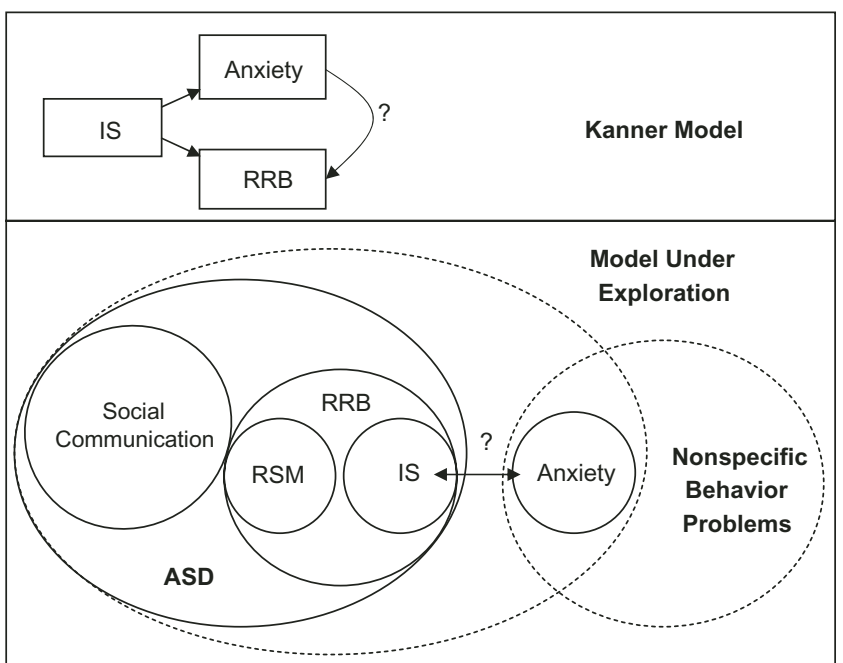

Figure 1. Models of anxiety-insistence on sameness (IS) relationship. ASD, autism spectrum disorder; RRB, restricted, repetitive behavior; RSM, repetitive sensory-motor behavior.

We also aim to evaluate the potential influence of cognitive level on anxiety, IS, and the relationship between them. The use of a large, carefully phenotyped genetic research sample of children with ASD allows us the statistical power to examine the Anxiety-IS association while controlling for age and verbal IQ, with the further benefit of assessing these target variables' relationships with ASD severity and other behavioral dimensions, such as irritability and aggression.

\section{Methods}

Participants

Data were collected as part of the Simons Simplex Collection (SSC), a genetic consortium study focusing on "simplex" families, in which a single child with ASD has no first-, second-, or third-degree relatives with suspected ASD (Fischbach \& Lord, 2010). SSC probands recruited from 12 university-based sites all met criteria for ASD on the Autism Diagnostic Observation Schedule (ADOS; Lord et al., 2000), Collaborative Programs for Excellence in Autism "ASD" criteria on the ADI-R (Rutter et al., 2003), and had a nonverbal mental age of at least 18 months (see https://sfari.org/funding/grants/abstracts/ simons-simplex-collection-ssc for detailed eligibility criteria). Children with marked motor impairments, blindness, deafness, genetic syndromes, or significant medical histories (e.g. very low birthweight) were excluded. Potential probands were also excluded if they had a sibling with language or psychological problems likely related to ASD.

Because the primary focus of the present study is the association between anxiety and IS symptoms, the sample described here was restricted to those SSC participants who had the CBCL 6-18 anxiety problems T-score, a normed summary score derived from the raw total of six items describing DSM-IV criteria for childhood anxiety disorders (Achenbach \& Rescorla, 2001). The final dataset included 1429 individuals aged 5 years 8 months to 18 years 0 months at the time of the in-person assessment (mean age $=10$ years 2 months; standard deviation $(\mathrm{SD})=3$ years 1 month); all children were 6 or older when the CBCL was administered. Mean verbal IQ was 80 $(\mathrm{SD}=31$, range $=5-167)$, and mean nonverbal IQ was 86 $(\mathrm{SD}=26$, range $=9-161)$.

Fourteen percent of the sample was female $(n=193)$. Ethnicities included 4\% African American ( $n=50$ individuals); 3\% Asian American $(n=49) ; 79 \%$ Caucasian $(n=1135) ; 8 \%$ more than one race $(n=114) ; 5 \%$ other $(n=60)$; and $1 \%$ not reported $(n=20)$. Ten percent of families reported Hispanic heritage $(n=145)$. Maternal education was a graduate or professional degree for $24 \%$ of the sample, $66 \%$ bachelor's degree or some college, and $10 \%$ high school degree or less.

\section{Procedures and Measures}

Children received an ADOS and cognitive testing (see Fischbach \& Lord, 2010 for IQ test hierarchy). Parents were interviewed using the ADI-R and the Vineland Adaptive Behavior Scales, Second Edition (Vineland-II; Sparrow, Cicchetti, \& Balla, 2005) and completed questionnaires about both the proband and the sibling, including the Social Responsiveness Scale (SRS; Constantino \& Gruber, 2005) and the CBCL. Parents provided informed consent, and procedures were approved by Institutional Review Boards at each university.

For a complete listing of SSC measures, see http:// sfari.org/sfari-initiatives/simons-simplex-collection/sscinstruments The primary measures used to group the sample in this study were (a) the ADI-R, a semistructured parent interview for the purpose of informing diagnosis of ASD, demonstrated to have good to excellent validity and reliability (Rutter et al., 2003). ADI-R items include current (Current) and historical (Ever) scores. Diagnostic algorithm domains of communication, reciprocal social interaction, and stereotyped and repetitive interests and behaviors are based on sums of specific item Ever scores. All examiners affiliated with the SSC achieved research reliability on the measure and maintained their reliability with semiannual checks throughout the data collection period. (b) The CBCL 6-18 is a parent-rated questionnaire with demonstrated reliability and validity that assesses a child's current social competence and emotional and behavior problems. A three-point scale $(0-2$, with higher scores indicating greater symptomatology) is used to rate 118 items. In addition to internalizing and externalizing subscales, eight DSM-IV syndrome scales are produced, 
including a six-item Anxiety Total Problems scale. All subscale raw totals correspond to T-scores based on the child's age and gender. Although the CBCL 6-18 has not been validated for use in the ASD population, its preschool equivalent (CBCL 1.5-5; Achenbach \& Rescorla, 2001) was found to have a factor structure and internal consistency similar to general population norms in a well-characterized sample of 128 young children with ASD (Pandolfi, Magyar, \& Dill, 2009).

\section{Design and Analysis}

CBCL Anxiety Problem T-scores were used to operationalize anxiety, as T-scores allowed sample grouping by clinical cut-offs (see below). Exploratory factor analysis of the ADI-R Stereotyped and Repetitive Behavior items, conducted in SPSS for Windows, Rel. 17.0.1 (2008) using promax rotation, confirmed an IS factor comprised of the items Difficulty with Minor Changes in Routine, Compulsions/Rituals, Resistance to Trivial Changes in the Environment, Abnormal Response to Specific Sensory Stimuli, Sensitivity to Noise, and Circumscribed Interests (CI) (see Supplementary Table SI), as well as an RSM factor (see Szatmari et al., 2006). Although the CI item has been interpreted as distinct from IS in previous literature (see Bishop et al., 2012), it was included in our IS factor because factor loadings, item-rest correlations, and alpha coefficients for this "Ever" item were similar to those statistics for the other five IS items. Distributions of IS factor scores were nearly identical to those of raw score sums for the IS-loading items, with scores of 2 and 3 collapsed to " 2 " per accepted use of the measure. A summed IS "Ever" raw total therefore was chosen to operationalize IS behaviors. Note that IS raw totals based on summed Current or Ever items were highly correlated $(r=0.85)$; results described later were identical when Current raw totals were used.

Distributions of and correlations between the anxiety and IS variables were generated in this sample of ASD participants. For one analysis only, data from 1184 nonASD siblings of the present sample participants (who were screened to exclude ASD characteristics but were not excluded on the basis of anxiety symptoms unless they had a history of hospitalization) were used to compare proband and sibling anxiety score distributions. IS data were not available for siblings.

In order to test the relationship between the anxiety and IS variables beyond bivariate correlation, groupings based on these variables were created in the ASD sample and assessed for cell size compared with expectations. Both the CBCL Anxiety T-scores and the IS "Ever" raw totals were divided and cross-tabulated: (a) at the sample medians; (b) at the highest and lowest $25 \%$ of each variable and the middle 50\%; and (c) at the CBCL Anxiety subclinical range of concern (T-scores of 65 and greater) against the 25-50-25 percent split of IS. As all methods yielded almost equivalent results in terms of observed vs. expected cell size discrepancy, we focus on the last method. The CBCL Anxiety T-score was divided into scores of 50-64 (54\%) and $65-80(46 \%)$, and the unnormed IS raw total was divided from 0 to $4(26 \%), 5$ to 8 (50\%), and 9 to $12(24 \%)$. One-way analysis of variance with Tukey post-hoc tests were used to assess group differences by chronological age and verbal IQ; linear regression modeling was used to test the effects of these variables on continuous anxiety T-scores and IS raw totals. For all statistical tests reported here, $P$-values were compared with a criterion standard of 0.001 to address multiplicity and to limit the likelihood of overinterpreting small effects in a large sample with many variables tested.

Next, behavioral phenotyping variables were assessed for potential effects of anxiety and IS. Controlling for chronological age and verbal IQ, multivariate general linear model (GLM) analyses were used to assess the effects of the continuous anxiety T-scores and IS raw totals on continuous and categorical dependent variables, including: (a) selected participant characteristics: gender, familial depression and anxiety disorders, and regression, seizure, speech delay, and current speech status; (b) ASD symptomatology: ADOS and ADI-R domain totals, ADOS severity scores (Gotham, Pickles, \& Lord, 2009), ADI-R RSM factor scores, SRS parent and teacher raw totals, Repetitive Behavior Scale-Revised (RBS-R; Bodfish, Symons, Parker, \& Lewis, 2000) subtotals, and clinician certainty ratings; (c) adaptive behavior: Vineland-II domain standard scores; and (d) nonspecific behavior and/or psychiatric problems: Aberrant Behavior Checklist (ABC; Aman, Singh, Stewart, \& Field, 1985) overall total and domain subtotals, CBCL subscale T-scores, ADOS and ADI-R specific item scores regarding aggression, overactivity, and anxiety.

\section{Results \\ Distributions of IS and Anxiety Scores}

Distributions of the variables used to operationalize anxiety and IS are shown in the online Supporting Information, Supplemental Figures S1 and S2. These variables had a small but significant correlation with each other in this ASD sample, $r=0.28(P<0.001) .{ }^{1}$ Forty-six percent of probands had CBCL Anxiety Problem T-scores in the

\footnotetext{
${ }^{1} \mathrm{~A}$ concern in interpreting the correlation of 0.28 between anxiety and IS as being statistically significant is that this relationship might be driven by the association of other covariates with each of these two variables. In order to partially address this issue, we considered the available covariates of verbal IQ, age, and gender. After adjusting for these three covariates, the partial correlation between anxiety and IS was 0.27 . Hence, the conclusion that there is a modest but statistically significant association between anxiety and IS remains.
} 


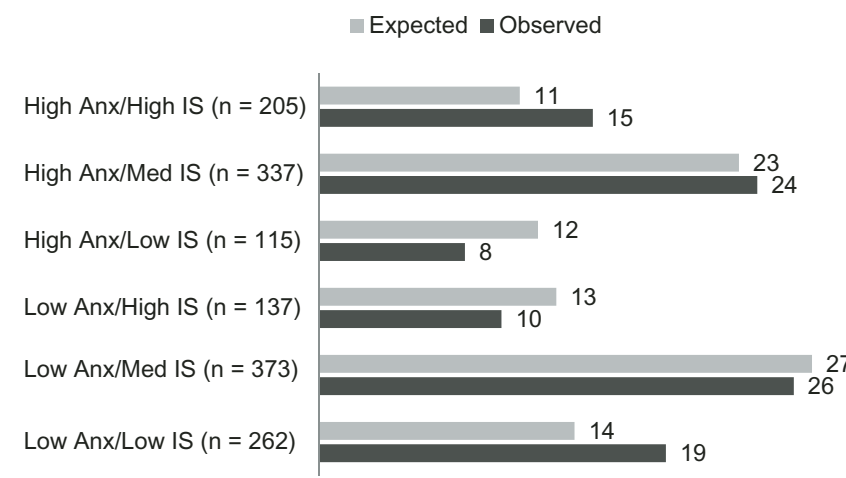

Figure 2. Expected by observed percentages in the anxietyinsistence on sameness (IS) groupings. Ns denote number of observed participants in each cell. Numbers following each bar denote the percentage of the total sample in each group (expected vs. observed).

subclinical range of concern and above (T-scores $\geq 65$ ) compared with only $9 \%$ of siblings who fell in this range for an odds ratio of 8.7. No other analyses presented here used sibling data.

As described earlier, the clinical vs. nonclinical CBCL Anxiety groups were cross-tabulated by the low, middle, and high IS groupings. All six groups had at least 100 individuals, supporting the finding that although anxiety and IS scores are correlated, they are relatively independent constructs. The largest discrepancies from expected group size occurred in the extremes: the proportions of children in the low anxiety $\times$ low IS group $(19 \%)$ or high anxiety $\times$ high IS group (15\%) were greater than the expected percentages for those groups (14 and 11\%, respectively). The proportions of children in the low anxiety $\times$ high IS group (10\%) and those in the high anxiety $\times$ low IS group (8\%) were lower than the expected percentages for those groups (13 and $12 \%$, respectively). See Figure 2 for a comparison of expected by observed percentages in all groups.

\section{Relationship of Covariates Age and Verbal IQ to IS and Anxiety}

In the binary $\mathrm{CBCL}$ clinical cut by $25-50-25$ IS variable groupings, chronological age did not approach significance for any comparison. Verbal IQ (VIQ) was significantly higher in the highest anxiety $\times$ highest IS group $(\mathrm{M}=89.2, \quad \mathrm{SD}=26.0)$ than it was for the lowest anxiety $\times$ lowest IS group $(\mathrm{M}=74.3, \mathrm{SD}=33.0)$ and lowest anxiety $\times$ middle IS group $(\mathrm{M}=76.8, \mathrm{SD}=32.3)$, respectively $(F(5,1423)=7.4, P<0.001$ for omnibus and both post-hoc results reported). Additionally, both chronological age and verbal IQ predicted CBCL Anxiety T-scores (age: $\mathrm{B}=0.02, \mathrm{SE}=0.01$; VIQ: $\mathrm{B}=0.04, \mathrm{SE}=0.01$ ) and IS raw totals (age and VIQ: $\mathrm{B}=0.01, \mathrm{SE}=0.002$ ) at the $P<0.001$ level, with greater age and VIQ associated with
Table I. Variables Significantly Predicted $(P<0.001)$ by Continuous Anxiety and/or Insistence on Sameness (IS) Scores

\begin{tabular}{lcccc}
\hline & & Anxiety T-scores & \multirow{2}{*}{ IS Raw Totals } \\
\cline { 3 - 3 } \cline { 5 - 5 } & Intercept (SE) & B(SE) & & B (SE) \\
\hline RBS-R Overall & $-3.4(10.2)$ & $0.7(0.2)^{*}$ & & n.s. \\
RBS-R Ritualistic & $-3.0(2.4)$ & $0.2(0.1)^{\text {a }}$ & & n.s. \\
RBS-R Sameness & $-5.3(3.6)$ & $0.2(0.1)^{*}$ & & n.s. \\
ABC Total & $-23.3(15.2)$ & $1.6(0.3)^{*}$ & & n.s. \\
ABC Irritability & $-12.8(5.2)$ & $0.5(0.1)^{*}$ & & n.s. \\
ABC Hyperactivity & $-0.03(6.5)$ & $0.5(0.1)^{*}$ & & n.s. \\
ABC Lethargy & $-10.9(4.5)$ & $0.3(0.1)^{*}$ & & n.s. \\
CBCL Internalizing & $3.6(4.2)$ & $0.8(0.1)^{*}$ & & n.s. \\
CBCL Externalizing & $19.7(6.4)$ & $0.7(0.1)^{*}$ & & n.s. \\
CBCL Thought & $47.7(5.1)$ & $0.3(0.1)^{*}$ & & n.s. \\
CBCL Social problems & $36.3(4.5)$ & $0.3(0.1)^{*}$ & & n.s. \\
CBCL Attention & $36.0(6.2)$ & $0.6(0.1)^{*}$ & & n.s. \\
CBCL Aggressive & $33.6(5.6)$ & $0.5(0.1)^{*}$ & & n.s. \\
CBCL ADHD & $38.0(5.1)$ & $0.4(0.1)^{*}$ & & n.s. \\
\hline
\end{tabular}

Note. Categorical groupings (anxiety clinical cut by IS 25-50-25 percent splits) had identical significant results with the following exceptions: neither anxiety nor IS categorical level predicted RBS-R Overall or CBCL thought subscales, and higher IS level significantly predicted RBS-R ritualistic subscale, intercept $B=5.4(S E=0.5)$, IS $B=1.4(S E=0.04)$.

* Significant at $P<0.001$.

${ }^{a}$ Approaching significance $(P=0.001)$.

$A B C$, Aberrant Behavior Checklist; ADHD, attention deficit hyperactivity disorder; $\mathrm{CBCL}$, Child Behavior Checklist; n.s., not significant at $P<0.001$; RBS-R, Repetitive Behavior Scale-Revised.

both higher anxiety and IS scores. Despite these significant findings, the effect sizes of age and verbal IQ on the continuous anxiety and IS variables appeared to be negligible (on CBCL Anxiety T-scores: $r=0.17, r^{2}=0.03$; on IS: $r=0.15, r^{2}=0.02$ ).

\section{The Role of Anxiety and IS in the ASD Phenotype}

Means and SDs for all dependent variables tested can be found in the online Supporting Information, Supplementary Table SII. Significant results of the GLM analyses using continuous CBCL Anxiety T-scores and IS raw totals are displayed in Table I, with a note summarizing the categorical findings.

The nonsignificant findings are of specific interest: IS raw totals were not significantly related to any of the variables assessed. Anxiety, too, had little association with participant demographic characteristics (including gender, race, and nonverbal IQ) or with most ASD diagnostic phenotyping measures (e.g. ADI-R and ADOS domains, SRS scores) or adaptive behavior (e.g. Vineland-II domain standard scores) in this sample. However, higher anxiety was associated with higher RBS-R Overall Total and Sameness subscale scores. Anxiety was also a significant predictor of a range of psychiatric and behavioral symptom scores, including $\mathrm{ABC}$ Overall, Irritability, 
Hyperactivity, and Lethargy totals, and CBCL Externalizing, Social Problems, Attention Problems, Thought Problems, and Aggressive Behavior totals. Neither anxiety nor IS was associated with parent diagnoses of anxiety or depressive disorders. No anxiety-by-IS interaction was significant for any dependent variable. Again, findings were similar when "Current" IS raw totals were used in place of "Ever" scores, and when 25-50-25 percent categorical splits were entered for both IS and anxiety variables (rather than the subclinical threshold cut for the CBCL Anxiety T-scores).

\section{Discussion}

Results of this study indicate that anxiety and IS function as distinct constructs, each with a wide range of expression in children with ASD across age and IQ levels. Anxiety was significantly more prevalent in probands with ASD than in the participants' non-ASD siblings (IS was not measured in these siblings and thus precluded comparison on that variable). Anxiety and IS continuous variables were not strongly associated with each other or with core autism diagnostic scores. Within this large ASD sample, both variables were minimally, although significantly, associated with participant chronological age and verbal IQ. More importantly, anxiety was associated with a variety of other psychiatric and behavioral symptoms in ASD, including irritability, attention problems, and aggression.

Despite the relatively low correlation between anxiety and IS scores in this sample $(r=0.28)$, high/high and low/low anxiety by IS groups were slightly more populated than would be expected if there was no relation between the variables. Additionally, anxiety scores were positively associated with the RBS-R Sameness subscale (see Bishop et al., 2012, for a discussion of the association between RBS-R and ADI-R RRB domains). Thus, the general conclusion that anxiety and IS are relatively independent within ASD is tempered by the reminder that some relationship exists. The discrepancy could be accounted for by different anxiety types within ASD, such as a heritable, moodrelated clinical anxiety vs. an RRB-specific "tenseness" associated with IS. That may suggest the notion of children with both high clinical anxiety and high IS tenseness having a "double-hit" that influences how they respond to their environment; however, we found no evidence of an anxiety-IS interaction in this study. Alternative models may involve mediators of the anxiety-IS relationship, such as level of sensory sensitivity or perseverative patterns to thoughts and behaviors. The relationship between anxiety and RRBs in general continues to warrant further exploration, particularly with regard to shared neurobiological mechanisms (Amaral, Schumann, \& Nordahl, 2008) and pharmacological implications.
Like anxiety, IS was not significantly associated with (other) core autism diagnostic features, such as scores on measures of social affect and reciprocity or RSM behaviors. Unlike anxiety (and the social communication and RSM behaviors themselves, based on previous literature; see Hus et al., 2007), IS was also independent from most participant characteristics and nonspecific behaviors tested here. Of particular interest, our findings replicate previous reports on the minimal association between IS and IQ (Cannon et al., 2010; Hus et al., 2007), and underscore the utility of IS behaviors as a means to characterize ASD samples that is independent of mediating variables.

One marked difference between anxiety and IS in this sample was the preponderance of strong associations between anxiety and other psychiatric and/or behavioral symptoms, such as inattention, hyperactivity, and social withdrawal, despite negligible associations between these symptoms and IS. It is unclear whether this is a meaningful profile or a measurement artifact. Because the anxiety groups were defined by a parent-rated CBCL subscale, it is possible that the associations between the anxiety ratings and $\mathrm{ABC}$ and $\mathrm{CBCL}$ subscales are driven by a parent-report effect and that the same patterns are not noted in IS or for the core autism measures because of clinician-mediated rating (i.e. ADI-R). However, the parent-rated anxiety scale was not predictive of the majority of parent-rated RBS-R subscales. Thus, anxious children with ASD indeed may have more behavioral problems than nonanxious children with ASD. Recent literature suggests that behavioral problems may be related to certain genetic profiles, such as children with 16p deletions (Hanson et al., 2010); thus, anxiety may be of value in genetic and neurobiological subgrouping despite, or in fact because of, these behavioral associations.

\section{Limitations}

Although the CBCL has shown some promise for use in ASD, it is not yet well validated in this population. The CBCL Anxiety scale likely bears some verbal ability effects that may influence these findings; this work should be replicated with specific anxiety measures (e.g. clinical interview or teacher report). Additionally, ADI-R IS "Ever" scores were chosen in order to include all children with a proclivity toward IS; although this represents a comparison of historical IS features with current anxiety symptoms, results were identical when ADI-R IS "Current" scores were used in place of "Ever." Of note, our IS factor included the item "Circumscribed Interests," which has not been included in several previous factor analyses of the ADI-R RRB domain (occasionally because of missing data); here, CI was deliberately included both to reflect empirical findings from the factor analysis and to include 
an item that involves fixed attention, a potential mechanism linking anxiety and IS symptoms. Finally, we chose a stringent significance criterion value to control for the quantity of statistical comparisons, which may have obscured some meaningful findings.

\section{Conclusions}

Parent-rated CBCL Anxiety T-scores and ADI-R IS raw totals appear to be minimally associated with each other and with chronological age and verbal IQ. Quantitative scores on continuous measures of anxiety and IS are likely to be relatively unrelated in children with ASD; thus, anxiety is another dimension on which large ASD samples could be stratified for genetic exploration. Neither anxiety nor IS was linked to (other) core autism features or severity scores. Anxiety was associated with a number of general behavioral symptoms, including attention problems and aggression. Describing research samples in terms of anxiety and IS variables may inform our understanding of mechanisms potentially common to both anxiety and ASD, such as "perseverative attention" in the form of CI in ASD and rumination in anxiety (Anholt et al., 2010; Sasson, Elison, Turner-Brown, Dichter, \& Bodfish, 2011).

\section{Acknowledgments}

This work was supported by grants from the Simons Foundation (SFARI) to K.G., A.B., and A.K., NIMH training grant (T32-MH18921; K. Gotham trainee) and NICHD Grants R01MH081873-01A1 and RC1MH089721 to C.L., and R01HD065277 to S.B. We are grateful to all of the families at the participating SFARI Simplex Collection (SSC) sites, as well as the principal investigators (A. Beaudet, R. Bernier, J. Constantino, E. Cook, E. Fombonne, D. Geschwind, D. Grice, A. Klin, D. Ledbetter, C. Lord, C. Martin, D. Martin, R. Maxim, J. Miles, O. Ousley, B. Peterson, J. Piggot, C. Saulnier, M. State, W. Stone, J. Sutcliffe, C. Walsh, E. Wijsman). We appreciate obtaining access to phenotypic data on SFARI Base. Approved researchers can obtain the SSC population dataset described in this study (https://ordering.base.sfari. org/ browse_collection/archive[sfari_collection_v9_1]/ ui:view) by applying at http://sfari.org/resources/ sfari-base/request-data-and-biospecimens We gratefully acknowledge the support of Ed Cook, Andrew Pickles, Devon Oosting, LeeAnne Green-Snyder, Amy Esler, Fiona Miller, Jennifer Olson, Susan Risi, Kathryn Larson, and Kathy Hatfield.

\section{References}

Achenbach, T.M., \& Rescorla, L. (2001). Manual for the ASEBA school-age forms \& profiles. Burlington, VT: ASEBA.
Aman, M.G., Singh, N.N., Stewart, A.W., \& Field, C.J. (1985). The Aberrant Behavior Checklist: A behavior rating scale for the assessment of treatment effects. American Journal of Mental Deficiency, 89, 485-491.

Amaral, D., Schumann, C., \& Nordahl, C. (2008). Neuroanatomy of autism. Trends in Neurosciences, 31, 137-145.

Amaral, D.G., Bauman, M.D., \& Schumann, C.M. (2003). The amygdala and autism: Implications from non-human primate studies. Genes, Brain, and Behavior, 2, 295-302.

Anholt, G.E., Cath, D.C., Oppen, P., Eikelenboom, M., Smit, J.H., et al. (2010). Autism and ADHD symptoms in patients with OCD: Are they associated with specific OC symptom dimensions or OC symptom severity? Journal of Autism and Developmental Disorders, 40, 580-589.

Balemans, M., Huibers, M., Eikelenboom, N., Kuipers, A.J., van Summeren, R., et al. (2010). Reduced exploration, increased anxiety, and altered social behavior: Autistic-like features of euchromatin histone methyltransferase 1 heterozygous knockout mice. Behavioral Brain Research, 208, 47-55.

Baron-Cohen, S., Ring, H.A., Bullmore, E.T., Wheelwright, S., Ashwin, C., \& Williams, S.C. (2000). The amygdala theory of autism. Neuroscience and Biobehavioral Reviews, 24, 355364.

Benno, R., Smirnova, Y., Vera, S., Liggett, A., \& Schanz, N. (2009). Exaggerated responses to stress in the BTBR T+tf/J mouse: An unusual behavioral phenotype. Behavioral Brain Research, 197, 462-465.

Ben-Sasson, A., Cermak, S.A., Orsmond, G.I., Tager-Flusberg, H., Kadlec, M.B., \& Carter, A.S. (2008). Sensory clusters of toddlers with autism spectrum disorders: Differences in affective symptoms. Journal of Child Psychology and Psychiatry, 49, 817-825.

Bishop, S.L., Hus, V., Huerta, M., Gotham, K., Duncan, A., et al. (2012). Subtypes of restricted and repetitive behaviors in children with autism spectrum disorders. Journal of Autism and Developmental Disorders, DOI 10.1007/s10803-012-1671-0

Bodfish, J.W., Symons, F.J., Parker, D.E., \& Lewis, M.H. (2000). Varieties in repetitive behavior in autism. Journal of Autism and Developmental Disorders, 30, 237-243.

Bolton, P.F., Pickles, A., Murphy, M., \& Rutter, M. (1998). Autism, affective and other psychiatric disorders: Patterns of familial aggregation. Psychological Medicine, 28, 385-395.

Cannon, D.S., Miller, J.S., Robison, R.J., Villalobos, M.E., Wahmhoff, N.K., \& Allen-Brady, K. (2010). Genome-wide linkage analyses of two repetitive behavior phenotypes in Utah pedigrees with autism spectrum disorders. Molecular Autism, 1, $1-13$.

Constantino, J.N., \& Gruber, C. (2005). The Social Responsiveness Scale. Los Angeles, CA: Western Psychological Services.

Cuccaro, M.L., Shao, Y., Grubber, J., Slifer, M., Wolpert, C.M., et al. (2003). Factor analysis of restricted and repetitive behaviors in autism using the Autism Diagnostic Interview-Revised. Child Psychiatry and Human Development, 34, 3-17.

DeLong, R.G., \& Dwyer, J.T. (1988). Correlation of family history with specific autistic subgroups: Asperger's syndrome and bipolar affective disease. Journal of Autism and Developmental Disorders, 18, 593-600.

Fischbach, G., \& Lord, C. (2010). The Simons Simplex Collection: A resource for identification of autism genetic risk factors. Neuron, 68, 192-195. 
Gadow, K.D., DeVincent, C., \& Schneider, J. (2008). Predictors of psychiatric symptoms in children with an autism spectrum disorder. Journal of Autism and Developmental Disorders, 38, 1710-1720.

Gadow, K.D., Roohi, J., DeVincent, C.J., \& Hatchwell, E. (2008). Association of ADHD, tics, and anxiety with dopamine transporter (DAT1) genotype in autism spectrum disorder. Journal of Child Psychology and Psychiatry, 49, 1331-1338.

Gotham, K., Pickles, A., \& Lord, C. (2009). Standardizing ADOS scores for a measure of severity in autism spectrum disorders. Journal of Autism and Developmental Disorders, 39, 693705.

Green, S., \& Ben-Sasson, A. (2010). Anxiety disorders and sensory over-responsivity in children with autism spectrum disorders: Is there a causal relationship? Journal of Autism and Developmental Disorders, 40, 1495-1504.

Hallett, V., Ronald, A., \& Happé, F. (2009). Investigating the association between autistic-like and internalizing traits in a community-based twin sample. Journal of the American Academy of Child and Adolescent Psychiatry, 48, 618627.

Hanson, E., Nasir, R.H., Fong, A., Lian, A., Hundley, R., et al. (2010). Cognitive and behavioral characterization of $16 \mathrm{p} 11.2$ deletion syndrome. Journal of Developmental and Behavioral Pediatrics, 31, 649-657.

Howlin, P., Mawhood, L., \& Rutter, M. (2000). Autism and developmental receptive language disorder - A follow-up comparison in early adult life. II: Social, behavioural, and psychiatric outcomes. Journal of Child Psychology and Psychiatry, 41, 561-578.

Hus, V., Pickles, A., Cook, E., Risi, S., \& Lord, C. (2007). Using the Autism Diagnostic Interview-Revised to increase phenotypic homogeneity in genetic studies of autism. Biological Psychiatry, 61, 438-448.

Joosten, A.V., Bundy, A.C., \& Einfeld, S.L. (2009). Intrinsic and extrinsic motivation for stereotypic and repetitive behavior. Journal of Autism and Developmental Disorders, 39, 521531.

Joshi, G., Petty, C., Wozniak, J., Henin, A., Fried, R., et al. (2010). The heavy burden of psychiatric comorbidity in youth with autism spectrum disorders: A large comparative study of a psychiatrically referred population. Journal of Autism and Developmental Disorders, 40, 1361-1370.

Kamp-Becker, I., Ghahreman, M., Smidt, J., \& Remschmidt, H. (2009). Dimensional structure of the autism phenotype: Relations between early development and current presentation. Journal of Autism and Developmental Disorders, 39, 557571.

Kanne, S.M., Abbacchi, A.M., \& Constantino, J.N. (2009). Multiinformant ratings of psychiatric symptom severity in children with autism spectrum disorders: The importance of environmental context. Journal of Autism and Developmental Disorders, 39, 856-864.

Kanner, L. (1943). Autistic disturbances of affective contact. Nervous Child, 2, 217-250.

Lesch, K., \& Mossner, R. (1998). Genetically driven variation in serotonin uptake: Is there a link to affective spectrum, neurodevelopmental, and neurodegenerative disorders? Biological Psychiatry, 44, 179-192.
Leyfer, O.T., Folstein, S.E., Bacalman, S., Davis, N.O., Dinh, E., \& Morgan, J. (2006). Comorbid psychiatric disorders in children with autism: Interview development and rates of disorders. Journal of Autism and Developmental Disorders, 36, 849861.

Lord, C., Risi, S., Lambrecht, L., Cook, E., Leventhal, B., et al. (2000). The Autism Diagnostic Observation ScheduleGeneric: A standard measure of social and communication deficits associated with the spectrum of autism. Journal of Autism and Developmental Disorders, 30, 205223.

Pandolfi, V., Magyar, C.I., \& Dill, C.A. (2009). Confirmatory factor analysis of the Child Behavior Checklist $1.5-5$ in a sample of children with autism spectrum disorders. Journal of Autism and Developmental Disorders, 39, 986-995.

Piven, J., \& Palmer, P. (1999). Psychiatric disorder and the broad autism phenotype: Evidence from a family study of multipleincidence autism families. American Journal of Psychiatry, 156, 557-563.

Rutter, M., Le Couteur, A., \& Lord, C. (2003). Autism Diagnostic Interview-Revised - WPS (WPS ed.). Los Angeles, CA: Western Psychological Services.

Sasson, N., Elison, J., Turner-Brown, L., Dichter, G., \& Bodfish, J. (2011). Brief report: Circumscribed attention in young children with autism. Journal of Autism and Developmental Disorders, 41, 242-247.

Silverman, W.K., Fleisig, W., Rabian, B., \& Peterson, R.A. (1991). Child Anxiety Sensitivity Index. Journal of Clinical Child Psychology, 20, 162-168.

Smalley, S.L., McCracken, J., \& Tanguay, P. (1995). Autism, affective disorders, and social phobia. American Journal of Medical Genetics, 60, 19-26.

Sparrow, S.S., Cicchetti, D.V., \& Balla, D.A. (2005). Vineland Adaptive Behavior Scales, 2nd ed. Circle Pines, MN: American Guidance Service, Inc.

SPSS for Windows, Rel. 17.0.1. Chicago, IL: SPSS, Inc.

Sukhodolsky, D.G., Scahill, L., Gadow, K.D., Arnold, L.E., Aman, M.G., et al. (2008). Parent-rated anxiety symptoms in children with pervasive developmental disorders: Frequency and association with core autism symptoms and cognitive functioning. Journal of Abnormal Child Psychology, 36, 117128.

Sweeten, T., Posey, D., Shekhar, A., \& McDougle, C. (2002). The amygdala and related structures in the pathophysiology of autism. Pharmacology, Biochemistry, and Behavior, 71, 449455.

Szatmari, P., Georgiades, S., Bryson, S., Zwaigenbaum, L., Roberts, W., et al. (2006). Investigating the structure of the restricted, repetitive behaviours and interests domain of autism. Journal of Child Psychology and Psychiatry, 47, 582590.

Takayanagi, Y., Fujita, E., Yu, Z., Yamagata, T., Momoi, M., et al. (2010). Impairment of social and emotional behaviors in Cadm1-knockout mice. Biochemical and Biophysical Research Communications, 396, 703-708.

Witwer, A., \& Lecavalier, L. (2010). Validity of comorbid psychiatric disorders in youngsters with autism spectrum disorders. Journal of Developmental and Physical Disabilities, 22, 367380 . 
Wood, J., Drahota, A., Sze, K., Har, K., Chiu, A., \& Langer, D. (2009). Cognitive behavioral therapy for anxiety in children with autism spectrum disorders: A randomized, controlled trial. Journal of Child Psychology and Psychiatry, 50, 224-234.

\section{Supporting Information}

Additional Supporting Information may be found in the online version of this article:
Figure S1. Distribution of Child Behavior Checklist (CBCL) 6-18 anxiety problems T-scores.

Figure S2. Distribution of insistence on sameness raw total scores. ADI-R, Autism Diagnostic Interview-Revised. Table SI. Factor Loadings for Autism Diagnostic Interview-Revised Restricted, repetItive, and Stereotyped Patterns of Behavior Domain, Promax Rotation.

Table SII. Phenotyping Variables by Anxiety and Insistence on Sameness Groups. 